\title{
Opportunities and challenges of Blockchain-Oriented systems in the tourism industry.
}

\author{
Fabio Caddeo \\ University of Cagliari \\ Cagliari, Italy \\ fabio.caddeo@yahoo.it
}

\author{
Andrea Pinna \\ Department of Mathematics and Computer Science \\ University of Cagliari \\ Cagliari, Italy \\ pinna.andrea@unica.it
}

\begin{abstract}
The tourism industry is increasingly influenced by the evolution of information and communication technologies (ICT), which are revolutionizing the way people travel. In this work we want to investigate the use of innovative IT technologies by DMOs (Destination Management Organizations), focusing on blockchain technology, both from the point of view of research in the field, and in the study of the most relevant software projects. In particular, we intend to verify the benefits offered by these IT tools in the management and monitoring of a destination, without forgetting the implications for the other stakeholders involved. These technologies, in fact, can offer a wide range of services that can be useful throughout the life cycle of the destination.

Index Terms-Blockchain, BOSE, Tourism industry, ETourism
\end{abstract}

\section{INTRODUCTION}

Nowadays, tourists are increasingly involved in creating the travel experience, becoming promoters of what they experience first-hand and consequently contributing to the improvement of a destination. In other words, they become protagonists of a process of co-creation of value that can affect both the destination in general and individual companies operating in the sector; examples are "Expedia", one of the largest worldwide Online Travel Agencies (or OTA), and "TripAdvisor", a travel platform that offers the opportunity of reviewing the tourist services that you have enjoyed on holiday (not just accommodations, restaurants, or airlines, but also experiences and attractions). In fact, through the involvement of their customers / travellers, they have managed to increase their importance in the travel industry. With the spread of the Internet, the world of tourism has undergone a first transformation, leading to the spread of the so-called etourism. More precisely, according to the definition provided by Buhalis (2003) [2], e-tourism means "the digitization of all processes and value chains in the tourism, travel, hospitality and catering sectors that allow organizations to maximize their efficiency and effectiveness". This situation has given a strong impetus to the tourism sector, as it has allowed the various destinations to exploit this technology to improve their attractiveness by implementing appropriate online communication strategies. Recently, however, we are witnessing the transition from e-tourism to smart tourism.
In truth, however, rather than smart tourism it would be more right to talk about smart destinations, which Lopez de Avila (2015) [6] defines as: "an innovative tourist destination built on a state-of-the-art technology infrastructure, which ensures the sustainable development of tourist areas, accessible to all, and which facilitates the visitor's integration with its environment, increasing the quality of the experience and improving the quality of life of residents". This definition clearly shows the importance of ICTs for their integration into the physical infrastructure of the destinations.

The use of information technologies is therefore an essential requirement of new forms of tourism. On the one hand, they help tourists in identifying and purchasing the tourist products they prefer, making them increasingly involved in co-creation and co-promotion of travel experiences, and on the other hand they allow suppliers of such products to make their offerings known around the world.

One of the problems arising from the use of new technologies in tourism is the lack of public confidence. Blockchain technology would seem to provide a solution, as the strengthening of trust represents one of the potential effects deriving from its application to tourism, especially in a period of great uncertainty like the one we are currently experiencing. The formation of trust in the hospitality industry, in fact, is still a little-known aspect, since it depends on the subjectivity of individual tourists, or rather on the risk that they are willing to accept in travel experiences. In the specific case of blockchain technology, the protocols on which it is based are structured in such a way as to ensure greater involvement by the various tourism stakeholders and thus improve their experience in the sector.

As highlighted by Porru et al. (2017) [14], the growing focus on the world of blockchain technology has led to the need to create tools for the development of specific software oriented towards it (called blockchain-oriented software, or BOS). In general, BOS is defined as a system that works through the implementation of a blockchain. An example is the Ethereum platform, which can be defined as the largest decentralized digital platform in the world that uses blockchain technology for the realization not only of transactions, but 
also of particular programs called smart contracts, ensuring security, reliability and transparency [10], [12]. More precisely, "Smart Contracts" are programs written in a programming language and registered in the blockchain that self-execute when certain predetermined conditions occur. Pinna et al. (2019) [13] define Smart Contracts as programs stored within the public register of Ethereum and associated with a particular blockchain address, aimed at implementing a logical sequence of steps according to some well-defined rules.

In this work we want to investigate the use of innovative IT technologies by DMOs, focusing attention on blockchain technology. In particular, we intend to verify the potential offered by these tools in the management and monitoring of a destination, without forgetting the implications for the other stakeholders involved. Such technologies, in fact, can offer a wide range of services that can be useful throughout the entire destination life-cycle. They represent an indispensable tool both to provide DMOs with different advantages from the management point of view (for example in monitoring or marketing) in order to achieve the sustainability objective [16], both to ensure that tourists and operators in the sector have a satisfactory experience.

The investigation discussed in this paper is guided by the following research questions.

RQ1: to what extent does the scientific community address the problems relating to the use of blockchain technology in tourism? and

RQ2: what is the state of the art of the practical use of blockchain technology in tourism and DMOs?

To answer the first question, we examined the scientific literature on the SCOPUS database, and we deepened the study by examining the content of the works more related to technical and socio-economic issues, the results of which are presented in Section II. To answer the second question, we collected information regarding the most relevant blockchainoriented projects for tourism, analyzing both the functionalities and the technology used. Section 3 presents the study of eleven software projects currently operational or under development. Through the study of the scientific literature of reference and documentation on specialized websites, it is intended to firstly evaluate the contribution of these technologies to tourism managers, by laying the foundations for subsequent implementations. Furthermore, the Section 4 of the paper allows us to discuss the two directions towards which the tourism industry is moving towards thanks to the use of blockchain technology, i.e disintermediation and coordinaion \& coopetition, as is arising from our investigation. Finally, Section 5 concludes the paper.

\section{LITERATURE REVIEW}

Although the scientific literature on the topic of blockchain is increasingly extensive, there are still few contributions that analyse it in relation to the tourist industry, but all are suitable for new insights. To confirm this, in Fig. I you can check the number of articles published (or added to the lists of papers in press) annually dealing with the issue of blockchain related to

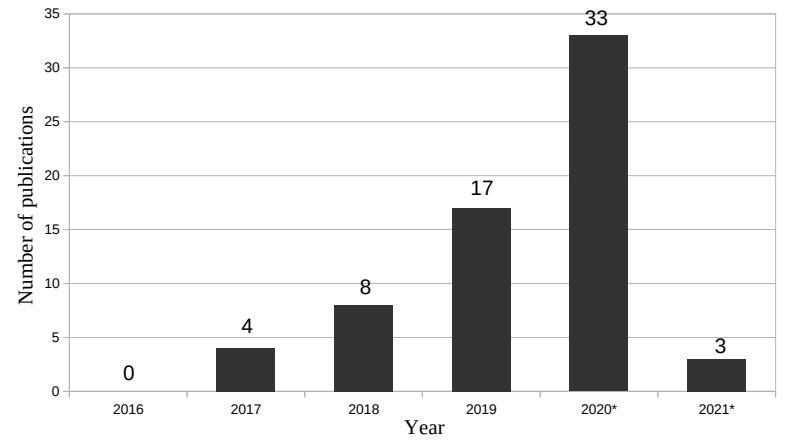

Fig. 1. Number of resulting publication per year by quering TITLE-ABS-KEY ("blockchain" AND "tourism") in SCOPUS. For the years 2020 and 2021, the symbol $*$ indicates the presence of incomplete data, updated to November 2020 .

tourism. The data were recovered in November 2020 through the use of the Scopus database, setting the search by query TITLE - ABS - KEY ("blockchain" AND "tourism") with which were extracted scientific papers containing the words "blockchain" and "tourism" in the title, in the abstract or among the keywords. The graph shows that since 2016, with no article published on this theme, the number has doubled from year to year, which highlights the growing interest of the scientific community towards the application of blockchain technology in the tourism sector.

\section{A. Selected literature}

The selection process consisted of the following steps. From the list of 65 papers obtained from the above described query, the papers whose abstracts dealt with the application of blockchain technology to tourism as the main topic of the study were selected. Of these, the availability of the full text was verified (either by publishers' websites and by courtesy of the authors). Then, we have focused our attention on the scientific contributions of the last two years in relation to the general principles of the BOSE [14]. In particular, we chose the papers that discussed the opportunities offered by blockchain technology applied in the tourism sector. We have also included the papers that discussed the challenges that the tourism industry will have to face with the adoption of blockchain technology, both from a technological point of view and from an economic-organizational point of view. Tab. II reports the summary of the scientific literature chosen and analysed in this work about the application of blockchain technology in tourism.

The analysis of the articles in Tab. I] aims to be an opportunity to present the general framework of studies that address the relationship between blockchain technology and the tourism sector, to identify what may be its contribution to the development of this industry. In our survey, the important contribution of Önder and Treiblmaier stands out. They are among the most prolific authors dealing with the issue of the application of blockchain technology in tourism and enrich the ideas of reflection on this issue. In 2018, Önder and Treiblmaier 
TABLE I

SUMMARY OF THE RESEARCH PAPERS ANALYZED IN THIS STUDY

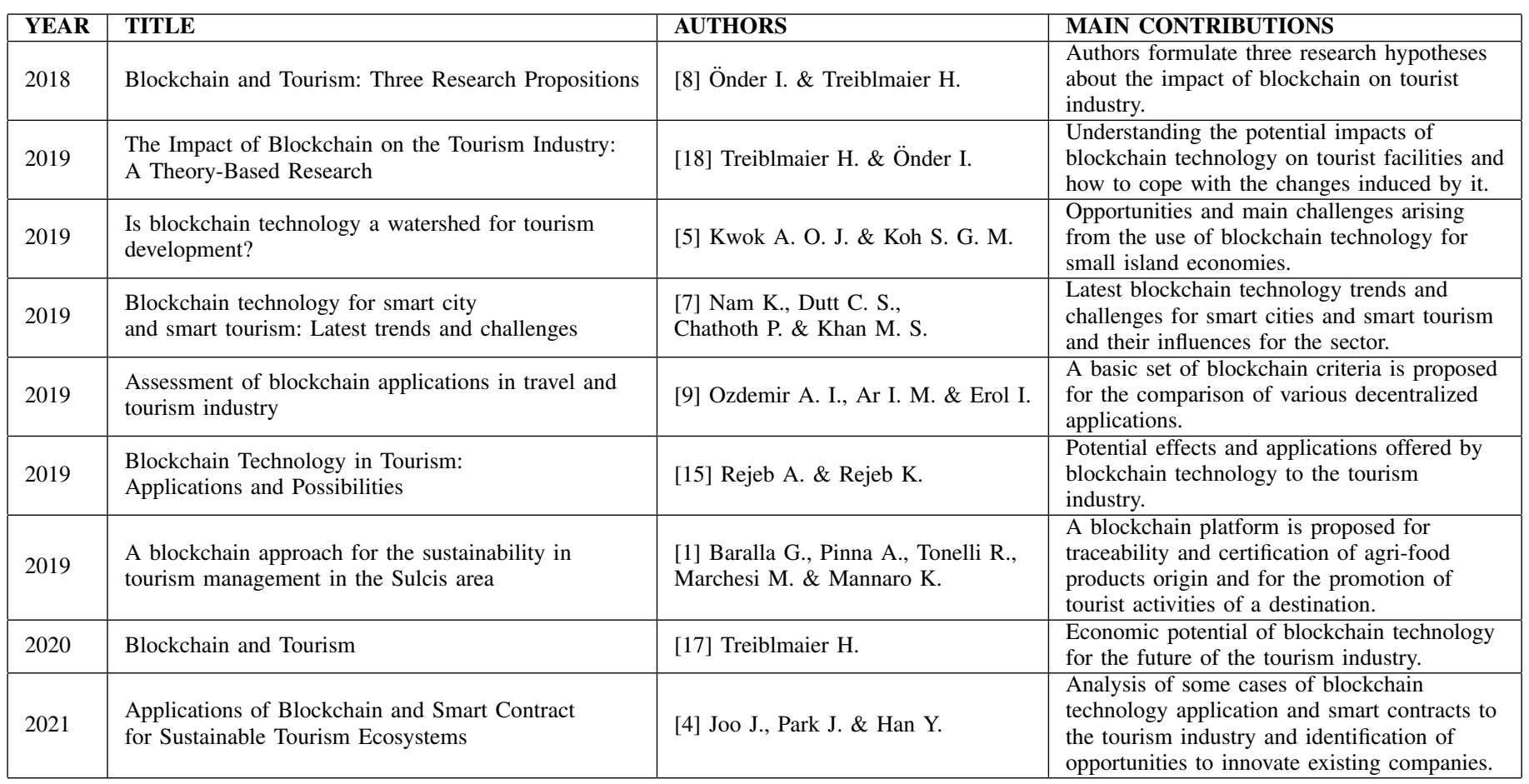

[8] conducted a study in which they formulated three research hypotheses concerning the impact of the blockchain on the tourist industry, claiming that this will bring: the activation of reliable systems for evaluating tourism products through the use of new forms of assessment and review technologies; the creation of new consumer-to-consumer (C2C) markets through the adoption of cryptocurrencies; disintermediation of the tourist industry. Before buying a trip and its related services online, it is normal for any traveller to first read the reviews of those who have already used the same service, considering this information more up-to-date and reliable than that given directly by travel service providers. Indeed, it is well known that online reviews of tourism products have a significant influence on potential consumers. However, these reviews are often subject to falsification by industry players themselves (such as hotel or restaurant owners). They seek to orient customers to their advantage by creating a not entirely truthful evaluation of their products by posting fake reviews; this is complicated by the fact that anyone can publish travel reviews without being asked for proof of having completed the tourist experience. To overcome this situation, and thus have correct online reviews, through blockchain technology it is possible to create evaluation systems that provide individuals with traceable identities through unique private keys, in order to avoid distortions in reviews and make them more reliable. This would create a decentralized, impartial, and transparent system that guarantees authentic and reliable reviews to potential travellers, which once entered in the blockchain register would no longer be possible to modify or delete. In such a system, would still be guaranteed the privacy of users, who could also be more encouraged in making reviews with any financial rewards in the form of tokens and cryptocurrencies to be received as a reward. Most tourism products often involve the transfer of money between partners located in different countries. This may require intervention by trusted intermediaries to conclude the transaction, with the consequence of having to pay additional large commissions; think for example of the $20 \%$ charged by Booking to hoteliers, overpricing which inevitably affects final consumers. Using cryptocurrencies, however, it is possible to ensure an easy exchange of money without the help of third parties intermediaries and participate in the emergence of new forms of $\mathrm{C} 2 \mathrm{C}$ markets of tourism products. Cryptocurrencies could therefore change the way tourists and operators exchange money and avoid the intervention of third parties (banks) in transactions, with consequent cost reductions. This is especially important where tourists' personal information, including financial data, cannot be entrusted to intermediaries. There are many systems that use blockchain to ensure high standards of security in transactions and at the same time greater protection of travellers' information. One of these is TripEcoSys (10), which aims to be the largest decentralized tourism platform in the world based on the Ethereum blockchain. It is a system in which it is possible to find in a single space all the providers of the different travel services (flights, accommodation, excursions, etc.) that normally the visitor should book independently of each other, and where you can earn cryptocurrency rewards by sharing your own experiences. Furthermore, given the characteristics 
of cryptocurrencies, through blockchain networks it is also possible to avoid the problem of converting foreign currencies and, consequently, further limit the influence exerted by intermediaries, making transactions safer. If, on the one hand, tourists would no longer have to convert money when travelling, thus eliminating all the risks associated with the exchange of foreign currencies, on the other hand companies would be free to adjust their prices according exchange rate variability. The advent of information technologies also in the tourism sector can therefore significantly reduce the intermediation chain towards the fruition of the tourist offer. The removal and replacement of intermediaries (also called disintermediation) is just one of the prerogatives of blockchain technology that in a short time will revolutionize the distribution and use of travel products and services, bringing significant benefits not only of an economic nature (cost savings). In general, it can be said that consumers will be more independent in organizing their travels. This disintermediation, however, does not only concern money transactions, but also directly affects the supply of tourism products. An initial form of disintermediation has already occurred with the advent of OTAs, but now also these could be replaced by blockchain-based, open source and decentralized travel platforms, such as WindingTree as discussed in the next section, which can eliminate the power exercised by intermediaries on the market. Another example is the Locktrip platform (see Table 2), which allows travellers to book hotels and other travel services without the intervention of any intermediary and, at the same, time provide feedback on the quality of the service. All this by using blockchain technology. From these simple examples, one can already understand the extent of the blockchain in the future of the tourist industry. In another study, Treiblmaier \& Önder [18] also set out to analyse the potential impacts of blockchain technology on tourism facilities and how organizations could cope with the changes it induces. In general, by interviewing the managers of ten European Destination Management Organizations (DMOs), they highlighted that blockchain can be an important resource for many tourist organizations because it has the potential to change market structures. However, this is still a complex technology as it requires a certain investment by organizations, but they may not have the necessary financial resources and appropriate know-how to exploit it adequately. It follows, therefore, that this technology can be a new (important) resource only for those organizations that are in a position to exploit it for their own benefit, especially in a sector such as tourism. In 2020, Treiblmaier [17] summarizes and discusses the current state of the art, describing a list of twelve use cases of blockchain adoption in tourism, and the most relevant theoretical aspects, according to the academic literature. It focuses attention on the economic aspects of tourism disintermediation, highlighting this use case as looming in the tourism sector, and as a challenge that the tourism organization must be ready to face.

Kwok and Koh (2018) [5] confirm the high potential of blockchain technology, analysing its opportunities in relation to the economies of small islands, which could exploit it to their advantage to compete with larger and often more renowned destinations. According to the two authors, in fact, blockchain technology represents a valid aid for such economies, strongly limited by their small size and their insularity, to implement tourism policies that encourage their economic growth. For example, Aruba is seeking to increase tourism revenue through the creation of a blockchain-based platform for travel bookings, while the Caribbean islands are promoting the adoption of their own regional cryptocurrency. In particular, the effects resulting from the application of the blockchain to small island tourism can be traced back to four categories that can create beneficial effects for both tourists and destinations. In summary, these are: the general offer of a better tourist experience to visitors; greater speed and security in transactions with foreign countries thanks to the use of cryptocurrencies; diversification of the financial offer and use of state-owned cryptocurrencies; finally, a reduction in costs for host destinations. All this, however, can only be possible if foreign tourists are able to understand it and if local operators are able to accept it, thus avoiding limiting its use to a restricted group of experienced users.

Nam et al. (2019) [7] analysed the latest trends and challenges regarding blockchain technology for smart cities and smart tourism, focusing their attention on the comparison of thirteen decentralized applications (Dapps) of the tourism industry. In particular, scholars have formulated some research proposals about its evolution and influence in the industry, coming to the conclusion that the adoption of blockchain technology, and especially Dapps, will lead in the future to the creation of new business models and new market structures. Although a limited number of solutions have been analysed, especially when compared to the totality of existing blockchain solutions, their analysis revealed three characteristics common to all Dapps: cost reduction, increasing adoption of cryptocurrencies and development of new all-encompassing ecosystems. More precisely, a market will be created with strong competition between new online travel platforms based on blockchain and those already existing on the market, that to survive will be forced to change their business models and adapt to new emerging trends. Furthermore, since it will not be easy to ensure the adoption of blockchain technology by travellers and other stakeholders, some operators may also provide for the granting of incentives for the use of cryptocurrencies (the greater the incentives, the faster the adoption of blockchain technology). This will therefore lead to the identification of some "dominant players", that is those platforms with the largest number of users, which will impose themselves on the market.

Ozdemir et al. (2019) [9] also made a comparison between several DApps, providing some practical examples of how these are used in tourism. In particular, they have focused, however, on proposing a basic set of blockchain technology criteria that may prove useful for evaluating decentralized applications. For this purpose, according to the authors, it is necessary to consider the governance model of the blockchain, the platform on which it is implemented, the type of consent, 
TABLE II

ACTIVE OR PROPOSED BLOCKCHAIN ORIENTED SOFTWARE SYSTEM FOR TOURISM INDUSTRY

\begin{tabular}{|l|l|l|l|l|}
\hline NAME AND URL & NATIONALITY & ACTIVITY STATUS & KIND OF APPLICATION & BLOCKCHAIN \\
\hline $\begin{array}{l}\text { (1) WindingTree } \\
\text { https://windingtree.com/ }\end{array}$ & Switzerland & Operating & Booking hotels and flights & Ethereum \\
\hline $\begin{array}{l}\text { (2) LockTrip } \\
\text { https://locktrip.com/ }\end{array}$ & Bulgaria & Operating & Booking hotels, holiday homes and flights & Ethereum \\
\hline $\begin{array}{l}\text { 3) FoodChain } \\
\text { https://food-chain.it/ }\end{array}$ & Italy & Operating & Traceability of food products & Quadrans \\
\hline $\begin{array}{l}\text { (4) Bagtrax } \\
\text { https://bagtrax.eu/ }\end{array}$ & United Kingdom & Operating & Baggage tracking & ND \\
\hline $\begin{array}{l}\text { (5) Yookye } \\
\text { https://yookye.com/it }\end{array}$ & Italy & Operating & $\begin{array}{l}\text { Organization of the holiday } \\
\text { (holiday home, services, experiences) }\end{array}$ & Ethereum \\
\hline $\begin{array}{l}\text { (6) DTCM Tourism 2.0 } \\
\text { https://dubai10x.ae/ }\end{array}$ & United Arab Emirates & Announcement & Check occupancy status of hotel in Dubai & ND \\
\hline $\begin{array}{l}\text { (7) WICKET } \\
\text { https://www.wicketevents.com/ }\end{array}$ & Italy & Operating & Ticketing & Ethereum \\
\hline $\begin{array}{l}\text { (8) Trippki } \\
\text { https://trippki.com/ }\end{array}$ & United Kingdom & Operating & Hotel booking & Ethereum \\
\hline $\begin{array}{l}9) \text { Travelchain } \\
\text { https://travelchain.io/ }\end{array}$ & Russia & Prototype & Travel ecosystem & ND \\
\hline $\begin{array}{l}\text { (10) TripEcoSys } \\
\text { https://www.tripecosys.com/ }\end{array}$ & United Kingdom & Prototype & Travel ecosystem & Ethereum \\
\hline $\begin{array}{l}\text { (11) Sardcoin } \\
\text { https://www.sardcoin.eu/ }\end{array}$ & Italy & Prototype & Smart coupon ecosystem & Hyperledger Fabric \\
\hline
\end{tabular}

the use of cryptocurrencies [], the use of smart contracts and finally tokens. They argue that understanding these elements is the key to understand and improve any blockchain application. For example, the identification of the governance model applied to the blockchain is of fundamental importance to determine its characteristics, as well as the type of platform on which it is implemented can affect its performance [11]. However, their considerations need further development, especially considering the sample of DApps analysed: some of these applications, in fact, seem to no longer exist.

The study conducted by Baralla et al. (2019) [1], however, aims at proposing a blockchain platform for traceability and certification of origin of Sardinian agri-food products and for the promotion of tourist activities in Sulcis area, in Sardinia, with a view to a sustainability. Thanks to the blockchain characteristics, in fact, it is possible to guarantee secure and transparent information and ensure that it reaches all the stakeholders involved. Following this logic, the system proposed by Baralla et al. makes use of a series of smart contracts to ensure traceability and provenance of products, giving tourist/consumer the opportunity to verify their authenticity and provide their own feedback by posting messages, photos, and videos. In this way, tour operators can simultaneously improve their services and enrich the tourist offer of the area. Although the proposed system is still in progress and limited to a finite territory, it is still very promising also for the future tourist management of much larger territories.

Finally, Joo et al. (2021) [4] focused their attention on the study of blockchain technology and related smart contracts in the sustainable tourism sector and on identifying the best opportunities to innovate existing companies, providing some application examples. They also confirm the evidence already highlighted by other scholars about the usefulness of the blockchain to improve the transparency and safety of travel operations, users' trust, and the reduction of transaction costs. The proposed examples can also help as a stimulus for tour operators who want to make their businesses more innovative.

\section{PRojects AND APPliCATIONS OF BLOCKCHAin TECHNOLOGY IN TOURISM}

\section{A. Relevant Business initiatives and Research Projects}

In Tab. II we report eleven blockchain oriented software that are used in the tourism industry. The list presented is the result of two considerations: there are some software that are cited as relevant examples in the reference literature, and others that have emerged from a specific research on the main uses of blockchain technology in the tourism sector. In addition to the name and their URL, for each of them are indicated: the nationality, the activity status (distinguishing the operating software, from the announcements and the prototypes), their main functionality and, where available, the type of blockchain technology used.

\section{B. Fields of application of blockchain technology}

Although some of them have already been mentioned previously, in the tourism and hospitality industry there may be many potential applications of blockchain technology, some of which may also integrate with each other. Some of the main applications of blockchain in tourism and those that could be implemented to add value to the sector are summarized below.

1) Inventory management: Blockchain technology can be used in an inventory management system which in the hospitality sector can refer to the number of rooms available in hotels or the number of seats available on airplanes. Specifically, it can provide information on availability and coverage rate by sharing it with the various interested stakeholders, thus replacing any Property Management System (PMS) and CRS, with the consequent removal of intermediaries and 
related expenses. An example of this is the Swiss platform WindingTree (1), which allows hoteliers and airlines to list their availabilities, and tourists to book them. Another example is given by "BedSwap", a project on which the German group TUI (Tourism Union International) is working for an effective management of the hotel rooms of its partners in the markets served by the company itself. The Dubai Department of Tourism and Commerce (6), on the other hand, in 2018 launched "Tourism 2.0", with the aim of making Dubai the world's first destination for global travel and events by 2020 . It is a system that allows you to check hotel occupancy and room rates, allowing other tour operators to prepare their offers more effectively for customers.

2) Traceability of food products: In an increasingly globalized tourism context, it is important to provide destinations with tools to ensure traceability in the supply of food chain products, especially in those sectors of food and wine tourism whose competitive advantage derives from the use of organic, local, and sustainable products. Here blockchain can be used to create a system that allows people to access data on the origin of food products and then reconstruct the path of products from the field to the table. In practical, this could be done by scanning a QR Code or directly barcodes of products through devices registered in the blockchain. For example, Foodchain (3) is an Italian system that, also using the Internet of Things (IoT), traces food products from origin to the final consumer using blockchain technology. The information made public by the participating companies is accessible to end consumers through a QR Code applied on the packaging of products. This helps to increase their confidence in companies given the possibility to check all the information on the product.

3) Baggage tracking: Despite the progress made by airlines in handling luggage, many travellers continue to experience problems with lost luggage, which leads to a loss of time and money for both passengers and airlines themselves. Blockchain technology is proposed as a solution in this sense since it allows the tracking of baggage, which can be monitored at various essential points, through the automatic entry of the data collected in a public register, thus also speeding up check-in and effectively reducing waiting times at the airport. For example, Bagtrax (4) uses a sensor which, once attached to suitcases, allows you to locate your luggage during transfers and to claim immediate compensation in case of loss. This system uses blockchain technology to make tracking sequences protected and guarantee the data security of all the actors involved in the service: passengers, airlines, airport managers and insurance companies.

4) Reservations and ticketing: In this case, the blockchain's usefulness lies in possible uses related to making reservations, for the issuance of tickets and to contrast the black market, for example by creating standard protocols allowing buyers to use their electronic wallets to prove ownership of the ticket. This includes WICKET (7), an app launched by an Italian startup that uses a special protocol based on blockchain technology to digitize tickets and limit the phenomenon of speculation. It uses the GET (Guaranteed Entrance Token) protocol, already used in the Netherlands and Singapore for the sale of tickets for sporting events or other events such as concerts, fairs and congresses. This year, however, following the pandemic that has affected the entire planet, was proposed to bathing establishments to allow bathers to book their place on the beach and pay directly online. The system associates the ticket with the buyer's phone number, generating a unique QR code in a special wallet, and will make it accessible to the user via an app to allow the latter to access the facility.

5) Travellers loyalty: Travel industry operators can benefit from the creation of dedicated loyalty programs that issue tokens as rewards to travellers. In fact, it has been demonstrated that companies that use such systems are able to obtain a competitive advantage over their competitors, reach new potential customers and improve the perception of the brand in their eyes, thus strengthening the link between travellers and destinations. The startup Loyyal has launched a blockchainbased platform through which companies can manage their loyalty programs by offering various types of reward systems. The Arab Group Jumeirah, in collaboration with Dubai Holding, uses this platform to improve the efficiency of its loyalty programs. Trippki (8), on the other hand, has devised a loyalty program to allow customers and companies in the tourism sector to enter directly into contact with each other, thus promoting the disintermediation of the sector. Specifically, customers are given some tokens (that are registered in the blockchain without expiration date) to stay in a certain hotel, thus ensuring the possibility to redeem them at any time.

6) Identity, credential management and privacy: In order to improve the sector safety and protect the privacy of travellers, we could think of the definition of a (digital) global traveller identity through a system that allows to determine unequivocally the identity of a person. At the same time, it is also possible finding a solution to the problem of identity theft that tourists often encounter. For example, it will be possible to add biometric information (especially fingerprints and facial recognition) to the other registered information thus also simplifying the work of hotels, that will have only to record in the blockchain the arrival and departure dates of guests, without having to report them to the police or other authorities. From this point of view, the telecommunications company of air transport SITA (Société Internationale de Télécommunications Aéronautiques) is studying how the use of virtual or digital passports can reduce document checks during passenger journeys, thus eliminating the need to possess various travel documents. Through a single token containing biometric data and other personal information, and stored on mobile or wearable devices, travellers can in fact be quickly identified only once by any authority.

\section{Technology}

Apart from their functionality in the tourism sector, there are two main blockchain technologies adopted: smart contracts and DApps.

1) Smart contracts: Their programmability and automated execution independently of human interference offers many 
potentials for the tourism industry. For example, these allow: the activation of immediate payment systems in transactions, thus facilitating collaboration between hotels and travel agencies; the allocation of rooms to guests via digital keys on the blockchain; airlines can facilitate flight insurance by paying automatically as agreed in case of delay or cancellation. Most of the applications mentioned as an example in this section use, in fact, different smart contracts in their systems.

2) dApps for smart tourism: Are in the process of defining projects for online review systems, travel planning, direct communication with property owners, personalized marketing. For example, the innovative Italian startup Yookye (5) aims to offer tourists travel proposals tailored by local experts on the needs and aspirations of users. On the basis of the preferences found, the local experts, making use of their territorial knowledge and artificial intelligence, then create some travel proposals from which the user can choose. Everything is made more reliable thanks to the blockchain technology, which guarantees transactions in total security. In this way, users enjoy a secure and reliable short-term property booking service, which combines traditional payment methods with the most famous cryptocurrencies and the YOOK token of the same platform.

3) Breaking technological barrier: Through the promotion of transparent transactions, blockchain technology guarantees a higher level of trust and security in online travel platforms, since all data will only be upgradable through a consensual agreement between all participants in the network. From this, it is clear that blockchain can be a valid tool for neutrality and objectivity in travel information systems. In this kind of system, in fact, customers/tourists feel more comfortable in sharing their travel experiences in a more open and sincere way. Examples include TravelChain (9) and WindingTree(1). The first is a blockchain-based travel business that rewards travellers for the transparent sharing of information regarding their experiences; the other, instead, is a decentralized and open-source platform that aims to find a solution to several problems in the travel industry (such as high commissions, obsolete technology, high entry barriers and lack of innovation), all linked to a high degree of centralization. This is a system in which transaction data is grouped into blocks and replicated among all participants to ensure greater transparency and control of the travel package. In this way, customers are able to ascertain the actual value of a tourism product and gain more control and power over the planning of their travel experience.

\section{Discussion}

The analysis carried out revealed two main effects that blockchain technology can bring to the tourism sector.

1) Disintermediation: The progressive removal of intermediaries has already been widely discussed. Today, travel agencies add a level of intermediation to the tourism chain, capable of generating end-to-end trust, that is, between the consumer (the tourist) and the tourist operator (i.e the DMO). Thanks to the presence of a trustless mechanism, blockchain technology makes the role of travel agencies accessory, that will have to review their role in the tourism system (as experts in the sector or as facilitators). This is a challenging opportunity for traditional travel agencies (which will be able to count on direct human relations). What has not been said, however, is that, specifically in the tourism sector, blockchain can lead to the replacement of Global Distribution Systems (GDS) that allow transactions between different service providers (for example hotels, airlines, travel agencies, etc.) and OTAs through systems that allow peer-to-peer communications and transactions.

2) Coordination and coopetition: Thanks to the blockchain technology it is possible to create systems that see the aggregation of different travel products and services in such a way as to reduce any inefficiencies and achieve greater coordination between the various proposals. The aforementioned Yookye platform (5), for example, brings together in a single system numerous services and utilities for tourists, simplifying the organization of their vacation. Its users, therefore, will no longer need multiple Apps, but can find and book accommodation, tours and other services in a single App. Similarly, Sardcoin (11), a project of the University of Cagliari, offers a platform for the sale of Sardinian tourist services based on blockchain technology. That typology of systems implements the concept of coopetition, that is the constructive and collaborative competition between companies from which both benefit: participating companies will be able to use the infrastructure to include services, integrate their offers or to set up tourism promotion strategies with other existing infrastructures.

\section{Conclusions}

On the basis of what has been said so far, it can be also said that tourism is one of the most promising sectors for the development of blockchain technology.

According to the results reported in this paper, we are able to answer the research questions which guided us in this investigation. In particular, for what concerns the first research question we can say that even if the number of the published research paper is still limited (to a total of 65 in the SCOPUS database), the number of published paper per year doubles every year. We examined the content of nine selected paper from which we can analyze the directions of scientific research in this domain. The second research question allowed us to explore the blockchain-oriented software projects for tourism. We reviewed 11 software projects, many of them operational, providing a classification of their core functionality and the related technological aspects.

However, regardless of what the scope of the investigation, some other important aspects need to be taken into account in further research. First of all, it is necessary to consider that the blockchain is just a collective term that synthesizes a series of different tools; in fact, there are numerous blockchain protocols that determine as many implementations. Secondly, it must be borne in mind that it is a constantly developing technology which involves from the simplest protocols that 
form the basis of it, up to real new applications. This progress is aimed above all at reducing the complexity of the technology regarding the difficulties of understanding (and consequently also of application). Finally, it must be said that, despite continuous progress, blockchain technology cannot be tied to a single use but lends itself to numerous purposes, even if sometimes conflicting with each other. In addition, it can also be combined with other technologies, such as the Internet of Things (IoT) or artificial intelligence, which integrate with each other to achieve increasingly innovative applications. This is confirmed by the blockchain technology application cases reported in this paper. This is the case of FoodChain (3) and Bagtrax (4), which integrate blockchain with the IoT to achieve their goals, or even of the startup Yookye (5), which combines blockchain technology and artificial intelligence tools.

In this work we highlighted the most relevant issues of the blockchain technology applied to the tourism industry. However, it has emerged that the uses of blockchain technology are limited to initiatives by individual companies, so we recommend destination managers to deepen and encourage its application in the tourist management of the territories. Researchers could conduct empirical studies to assess the design of comprehensive systems that help managers in promoting this innovative tool.

\section{REFERENCES}

[1] Baralla, G., Pinna, A., Tonelli, R., Marchesi, M., Mannaro, K. (2019). "A blockchain approach for the sustainability in tourism management in the Sulcis area". In C. Gargiulo \& C. Zoppi (Eds.), Planning, nature and ecosystem services (pp. 904-919). Naples: FedOAPress. ISBN: 978-886887-054-6, DOI: 10.6093/978-88-6887-054.6

[2] Buhalis, D. (2003). "eTourism: Information Technology for Strategic Tourism Management". Pearson Education Limited, Harlow.

[3] Gretzel, U., Sigala, M., Xiang, Z., Koo, C. (2015) "Smart tourism: foundations and developments". Electron Markets 25, 179-188, DOI: 10.1007/s12525-015-0196-8

[4] Joo, J., J. Park, and Y. Han. (2021) "Applications of Blockchain and Smart Contract for Sustainable Tourism Ecosystems", in Evolutionary Computing and Mobile Sustainable Networks. Lecture Notes on Data Engineering and Communications Technologies, vol 53, pp. 773-780, DOI: 10.1007/978-981-15-5258-8_71

[5] Kwok, Andrei O.J., Sharon G.M. Koh. (2019) "Is blockchain technology a watershed for tourism development?", Current Issues in Tourism 22.20: 2447-2452, DOI: $10.1080 / 13683500.2018 .1513460$

[6] Lopez de Avila, A. (2015) "Smart destinations: XXI century tourism". ENTER2015 Conference on Information and Communication Technologies in Tourism, Lugano, Switzerland, February 4-6, 2015.

[7] Nam, K., Dutt, C. S., Chathoth, P., Khan, M. S. (2019). "Blockchain technology for smart city and smart tourism: Latest trends and challenges". Asia Pacific Journal of Tourism Research, 0(0), 1-15. DOI: https://doi.org/10.1080/10941665.2019.1585376

[8] Önder, I., Treiblmaier, H. (2018). "Blockchain and Tourism: Three Research Propositions". Annals of Tourism Research, Volume 72, pp. 180-182. ISSN 0160-7383, DOI: https://doi.org/10.1016/j.annals.2018.03.005.

[9] Ozdemir, A. I., Ar, I. M., Erol, I. (2020). "Assessment of blockchain applications in travel and tourism industry". Quality and Quantity, vol. 54, no. 5-6, 2020, pp. 1549-1563. doi:10.1007/s11135-019-00901-w

[10] Pierro, G. A, Tonelli R., Marchesi. M. (2020). ”An Organized Repository of Ethereum Smart Contracts. Source Codes and Metrics." Future internet 12.11 (2020): 197. DOI: 10.3390/fi12110197

[11] Destefanis, G., Ortu, M., Bowes, D., Marchesi, M., Tonelli, R. (2018). "On measuring affects of github issues' commenters." Proceedings of the 3rd International Workshop on Emotion Awareness in Software Engineering. 2018. DOI: $10.1145 / 3194932.3194936$
[12] Fenu, G., Marchesi, L., Marchesi, M., Tonelli, R. (2018). "The ICO phenomenon and its relationships with ethereum smart contract environment". In 2018 International Workshop on Blockchain Oriented Software Engineering (IWBOSE) (pp. 26-32). IEEE. DOI: 10.1109/IWBOSE.2018.8327568

[13] Pinna, A. Ibba, S. Baralla, G. Tonelli, R., Marchesi, M. (2019). "A Massive Analysis of Ethereum Smart Contracts. Empirical Study and Code Metrics". IEEE Access, DOI: 10.1109/ACCESS.2019.2921936

[14] Porru, S., A. Pinna, M. Marchesi, R. Tonelli. (2017) "BlockchainOriented Software Engineering: Challenges and New Directions," IEEE/ACM 39th International Conference on Software Engineering Companion (ICSE-C), Buenos Aires, pp. 169-171, DOI: 10.1109/ICSEC.2017.142

[15] Rejeb, A., Rejeb, K. (2019). "Blockchain Technology in Tourism: Applications and Possibilities". World Scientific News, volume 137, pp. 119-144, Available at SSRN: https://ssrn.com/abstract=3480457

[16] Pinna A., Baralla G., Marchesi M., Tonelli R. (2021). "Raising Sustainability Awareness in Agile Blockchain-Oriented Software Engineering". In 2021 International Workshop on Blockchain Oriented Software Engineering (IWBOSE). DOI: 10.1109/SANER50967.2021.00088

[17] Treiblmaier H. (2020). "Blockchain and Tourism". In: Xiang Z., Fuchs M., Gretzel U., Höpken W. (eds) Handbook of e-Tourism. Springer, Cham. DOI: https://doi.org/10.1007/978-3-030-05324-6_28-1

[18] Treiblmaier, H., Önder, I. (2019). "The Impact of Blockchain on the Tourism Industry: A Theory-Based Research Framework". In Business Transformation through Blockchain - Volume II (pp. 3-21). DOI: http://doi-org-443.webvpn.fjmu.edu.cn/10.1007/978-3-319-99058-3_1

[19] World Economic Forum. (2017) "Digital Transformation Initiative. Aviation, Travel and Tourism Industry", White paper 